\title{
Adaptive Power Control Using Large Scale Antenna of the Massive MIMO System in the Mobile Communication
}

\author{
Chang-Bin Ha ${ }^{1}$, Byung-Jun Jang ${ }^{2}$ and Hyoung-Kyu Song ${ }^{1 *}$ \\ ${ }^{1}$ uT Communication Research Institute, Sejong University \\ Seoul, South Korea \\ ${ }^{2}$ Department of Electrical Engineering, Kookmin University \\ Seoul, South Korea \\ [e-mail: songhk@sejong.ac.kr] \\ *Corresponding author: Hyoung-Kyu Song
}

Received March 4, 2015; accepted May 30, 2015; published August 31, 2015

\begin{abstract}
Although the massive MIMO system supports a high throughput, it requires a lot of channel information for channel compensation. For the reduction of overhead, the massive MIMO system generally uses TDD as duplexing scheme. Therefore, the massive MIMO system is sensitive to rapidly changing fast fading in according to time. For the improvement of reduced SINR by fast fading, the adaptive power control is proposed. Unlike the conventional scheme, the proposed scheme considers mobility of device for adaptive power control. The simulation of the proposed scheme is performed with consideration for mobility of device. The result of the simulation shows that the proposed scheme improves SINR. Since SINR is decreased in according to the number of device in the network by unit of cell, each base station can accommodate more devices by the proposed scheme. Also, because the massive MIMO system with high SINR can use high order modulation scheme, it can support higher throughput.
\end{abstract}

Keywords: Mobile communication, massive MIMO system, mobility, fast fading, power control, SINR

A preliminary version of this paper was presented at ICONI 2014, and was selected as an outstanding paper. This research was supported by Basic Science Research Program through the National Research Foundation of Korea(NRF) funded by the Ministry of Science, ICT and future Planning(No. 2013R1A2A2A01067708) 


\section{Introduction}

Recently, because of the exponential increase of mobile device, high throughput for network by unit of cell is demanding [1-2]. As suitable system for the demand, there are the single-user (SU) - multiple input multiple output (MIMO) and the multi-user (MU)-MIMO system [3-6]. The SU-MIMO system can simultaneously transmit multiple information to single user in according to the number of antenna. Therefore, the SU-MIMO theorectically supports proportionally increased throughput in according to the number of antenna. But, the MU-MIMO system simultaneously transmits multiple information to multiple users. Although the MU-MIMO system doesn't enhance throughput of each user, it supports high throughput for network by unit of cell.

Due to the advantages of the MIMO systems, the MIMO systems are included in wireless communication standards such as 3GPP long term evolution (LTE), IEEE 802.11x. However, because the MIMO systems simultaneously transmit multiple signals, the transmitted signals from other antennas affects as interference signal to each other. Therefore, the conventional MIMO systems such as the SU-MIMO and MU-MIMO system don't have proportionally high throughput in according to the number of antenna.

For proportionally high throughput in according to the number of antenna, in contrast with the conventional MIMO systems using a few antennas, the massive MIMO system using large scale antenna is emerging [8-11]. Because it effectively compensates interference signals and noise using large scale antenna, it supports theoretical throughput in according to the number of antenna compared to the conventional MIMO systems. As another advantage of the massive MIMO system, although the massive MIMO system uses linear pre-coding scheme and linear detection scheme such as zero forcing (ZF) and matched-filter (MF) with low complexity, it has high reliability in contrast with the conventional MIMO systems [11-14].

In the following two sections, the massive MIMO system model and the problems to be solved by the proposed scheme in the paper are described. Next, the proposed scheme and simulation result of the proposed scheme are specifically described. From the simulation result, the positive effects by the proposed scheme in the massive MIMO system are confirmed. The final section represents the conclusion of this paper.

\section{System Model}

The massive MIMO system is composed of the base station (BS) with $N$ antenna and $K$ mobile devices. In the massive MIMO system, it is assumed that each mobile device has single antenna. Also, in this paper, it is assumed that MF scheme is used as pre-coding scheme and detection scheme.

Fig. 1 and Fig. 2 show the downlink and uplink structure of the massive MIMO system, respectively. At Fig. 1, $\mathbf{F}$ means pre-coding matrix, $\mathbf{H}$ means entire channel between each device and each antenna at the BS. The pre-coding matrix based on MF is equal to Hermitian matrix of $\mathbf{H}$. The received signal at the BS is expressed as follows,

$$
\mathbf{y}_{B S}=\sum_{k=1}^{K}\left(\sqrt{P_{K}} \mathbf{H}_{k} s_{k}+n_{k}\right) .
$$


The received signal at the $k$-th device is expressd as follows,

$$
\mathbf{y}_{k}=\sqrt{P_{k}} \mathbf{F}_{k} \mathbf{H}_{k} s_{k}+\sum_{i=1, i \neq k}^{K}\left(\sqrt{P_{i}} \mathbf{H}_{i} \mathbf{F}_{i} s_{i}\right)+\mathbf{N}
$$

where $P_{k}$ is the transmission power for the $k$-th device, $\mathbf{H}_{k}$ is the channel between the $k$-th device and each antenna at the BS, $\mathbf{F}_{k}$ is the pre-coding matrix for the $k$-th device, $s_{k}$ is the signal for the $k$-th device, $\mathbf{N}$ is the independently generated noise with zero mean and white Gaussian distribution at the received signal.

When the received signal at the $k$-th device is detected, the equation for signal to noise plus interference ratio (SINR) of the $k$-th device is expressed as follows,

$$
\operatorname{SINR}_{k}=\frac{P_{k}\left\|\mathbf{F}_{k} \mathbf{H}_{k}\right\|^{2}}{\sum_{i=1, i \neq k}^{K}\left(P_{i}\left\|\mathbf{F}_{k} \mathbf{H}_{i}\right\|^{2}\right)+\sum_{i=1}^{K}\left(\left\|\mathbf{F}_{k} n_{i}\right\|\right)}
$$

where $n_{i}$ is generated noise at the $i$-th device.

This paper considers mobile communication with mobile device on pedestrian, bicycle, and automobile. The velocity of pedestrian, bicycle, and automobile is assumed as $3 \mathrm{~km} / \mathrm{h}$, $10 \mathrm{~km} / \mathrm{h}$, and $60 \mathrm{~km} / \mathrm{h}$, respectively.

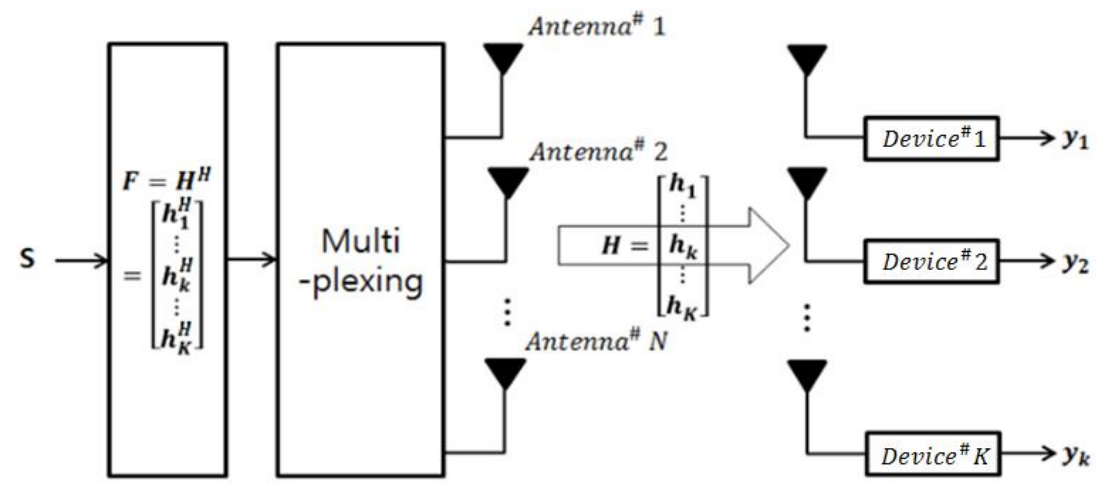

Base station

Fig. 1. Downlink model of massive MIMO system

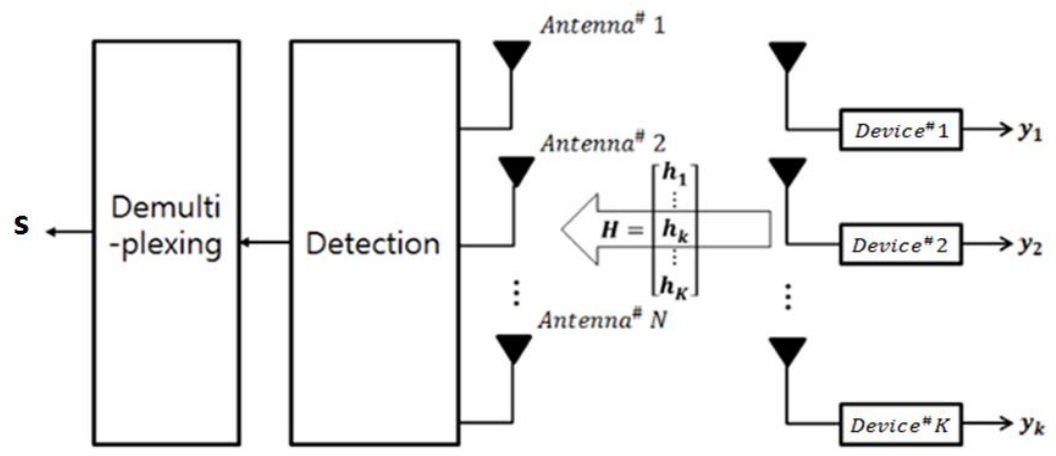

Base station

Fig. 2. Uplink model of massive MIMO system 
As shown at Fig. 1 and Fig. 2, the uplink and downlink channel of the massive MIMO system is same. The reason is that time division duplexing (TDD) is used as duplexing scheme in the massive MIMO system.

2.

The system parameters are shown in Table 1 and the traffic parameters are shown in Table

Table 1. System parameters

\begin{tabular}{|c|c|c|}
\hline System & Parameters & Value \\
\hline \hline \multirow{3}{*}{ OFDM [15-16] } & Carrier frequency $\left(f_{c}\right)$ & $100 \mathrm{GHz}$ \\
\cline { 2 - 3 } & Subcarrier spacing $(\square f)$ & $13 \mathrm{KHz}$ \\
\cline { 2 - 3 } & The number of subcarrier $\left(N_{c}\right)$ & 256 \\
\hline \multirow{3}{*}{ Massive MIMO } & The number of antenna at the BS $(N)$ & 200 \\
\cline { 2 - 3 } & The number of device with single antenna $(K)$ & $10: 10: 100$ \\
\cline { 2 - 3 } & Maximum transmission power & $23 \mathrm{dBm}$ \\
\hline Modulation scheme & 16 QAM \\
\hline
\end{tabular}

Table 2. Traffic parameters

\begin{tabular}{|c|c|}
\hline Traffic & Value \\
\hline \hline Path loss equation & $128.1+37.6 \cdot \log _{10}(\mathrm{R}) d B, \mathrm{R}$ in kilometers [17] \\
\hline \hline Cell coverage & $100 \mathrm{~m}$ \\
\hline Path number & 7 \\
\hline
\end{tabular}

\section{Problems to be Solved by the Proposed Scheme}

The massive MIMO system uses pilots for channel estimation. Because the massive MIMO system uses large scale antenna, it has many overhead compared to the conenvtional MIMO systems. For the reduction of overhead, the massive MIMO system uses TDD as duplexing scheme. Fig. 3 shows communication procedure of massive MIMO system based on TDD. As shown at Fig. 3, the estimated channel information at the certain $T_{\text {pilot }}$ is used for channel compensation until the next $T_{\text {pilot }}$. In Eq. (3), theorectically, for effective channel compesnation by the pre-coding, all pilots for channel estimation should be orthogonal to each other. However, because of the short coherence time, the non-orthgonal pilot is used in reality [18-20]. Therefore, channel estimation of the massive MIMO system is not perfect.

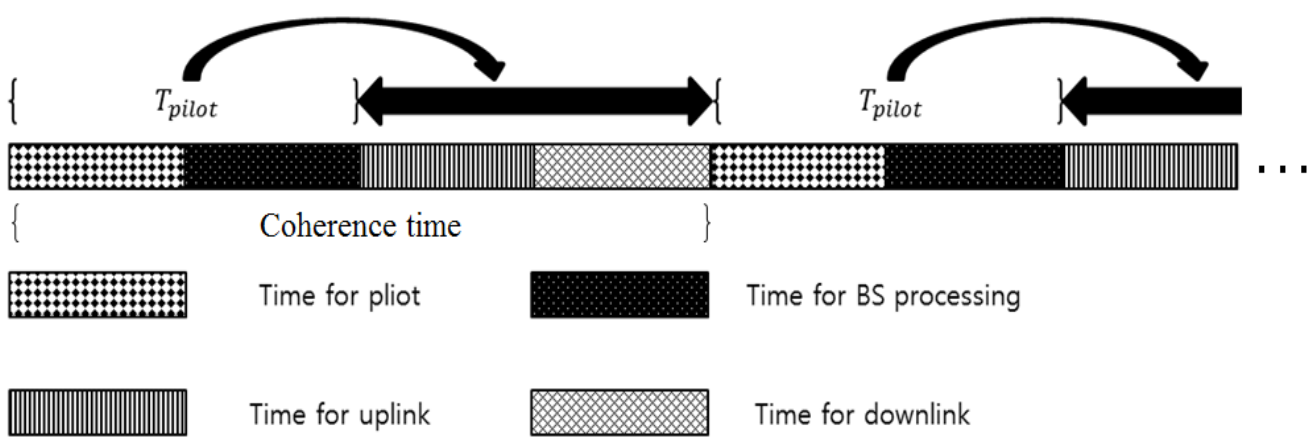

Fig. 3. Communication procedure of massive MIMO system 
Additionally, in the mobile communication system, fast fading is caused by the mobile device. The power of the received signal in the massive MIMO system is continually fluctuated in according to time by the fast fading. But, the pre-coding is generated based on the power of the received pilots at the $T_{\text {pilot }}$ during coherence time that assumes static channel. Therefore, if the power of the received signal is deviated from the power of the received pilot at the $T_{\text {pilot }}$, the channel compensation is not perfect.

\section{Adaptive Power Control Using Mobility of Device}

For enhacement of non-perfect channel compensation by the fast fading, the adaptive power control using mobility of device is proposed. In the massive MIMO system, the channel compensation is performed by the channel information that is estimated by the pilot during coherence time including the time for uplink and downlink. However, the coherence time is longer than the change cycle of the fast fading. Because the received signal at the BS is continually flcutated in according to time during the coherence time, the channel compensation using the estimated channel by the pilot is non-perfect. Therefore, in order to effectively comepnsate channel, power of the received signals at the each device should be equal to the estimated power by the pilot.

The core point of the proposed adaptive power control is to use the relationship between the fast fading and the Doppler frequency by the mobility of device. The fast fading has other characteristics such as mean and deviation of loss in according to the relative velocity between the BS and the mobile device. Fig. 4 shows the 10,000 samples of fast fading in according to velocity in corresponding to the pedestrian, bicycle, and automobile. As shown at Fig. 4, the attenuation degree by the fast fading is rapidly changed as relative velocity between the BS and the mobile device.

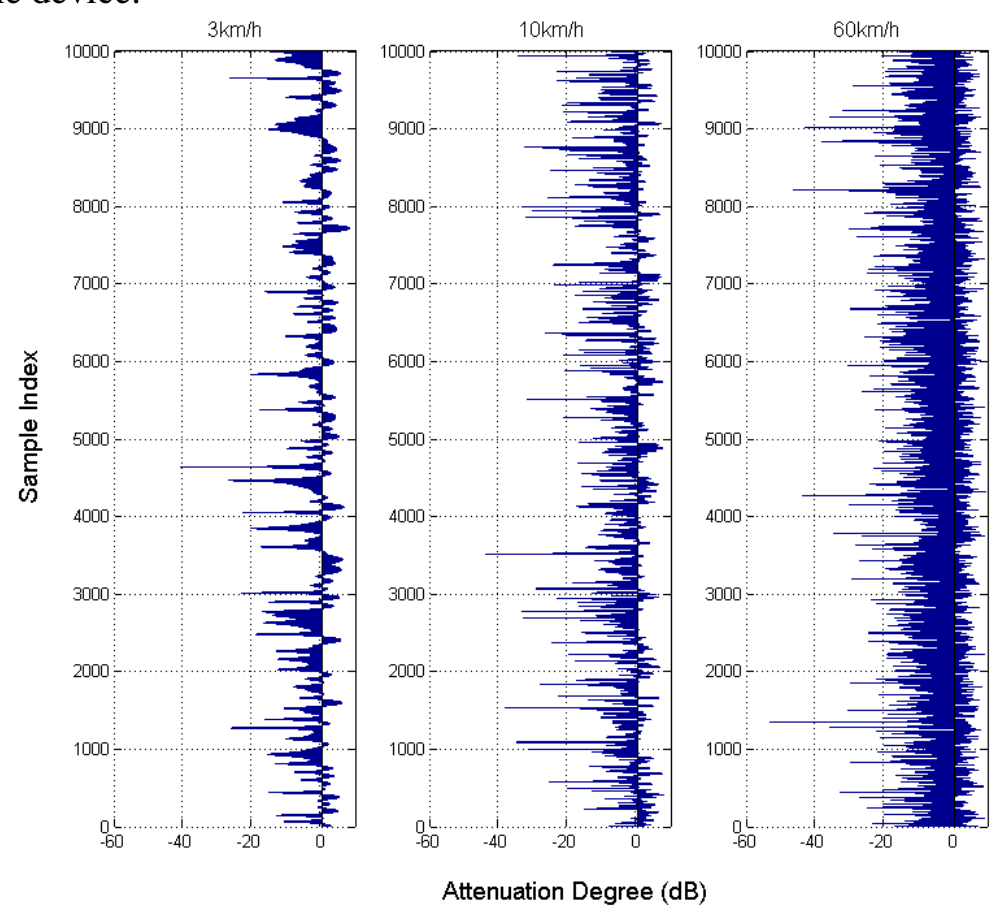

Fig. 4. The samples of fast fading in according to the velocity 
The Doppler frequency has large absolute value in according to the relative velocity between the transmitter and the receiver. In this paper, Doppler frequency is estimated by the maximum likelihood (ML) scheme [21]. The estimated Doppler frequency is the normalized Doppler frequency by the spacing between each subcarriers ( $\square f$ ) in the orthogonal frequency division multiplexing (OFDM). The normalized Doppler frequency in according to the relative velocity and the subcarrier spacing of the OFDM is induced as follows,

$$
\begin{gathered}
f_{d}=\frac{v \cdot f_{c}}{c} \\
f_{n d}=\frac{f_{d}}{\square f}
\end{gathered}
$$

where $v$ is the relative velocity between the BS and the device, $c$ is the velcotiy of the light, $f_{d}$ is the Doppler freuqency, and $f_{n d}$ is the normalized doppler frequency by the subcarrier spacing.

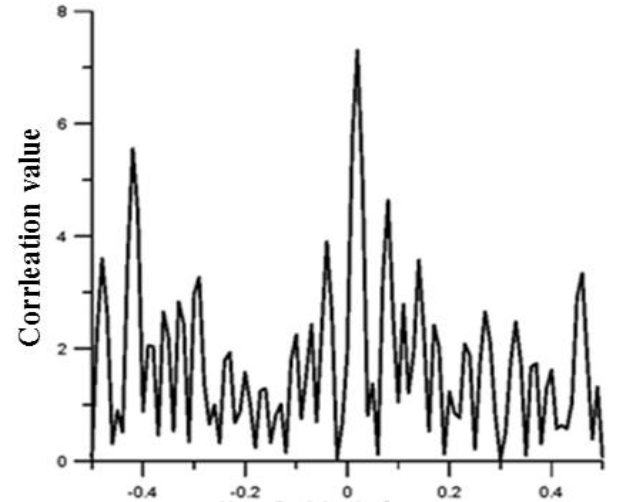

Normalized doppler frequency

$\mathbf{N}=\mathbf{3 0}$

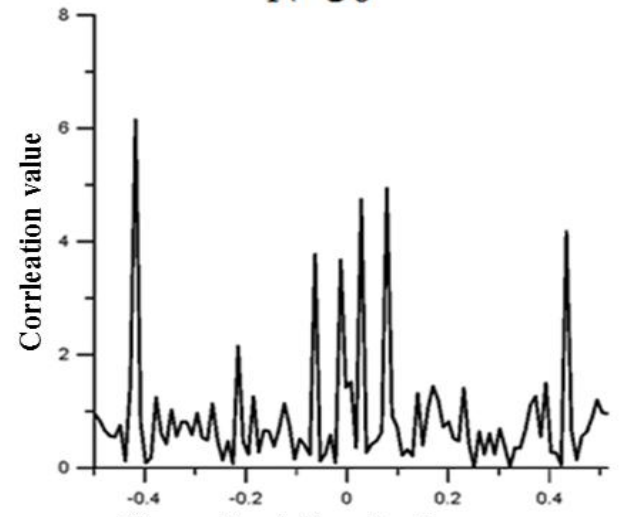

Normalized doppler frequency

$\mathbf{N}=\mathbf{9 0}$

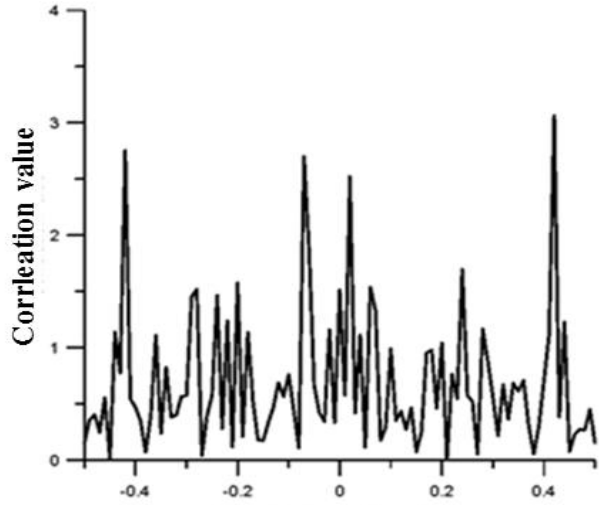

Normalized doppler frequency

$\mathbf{N}=60$

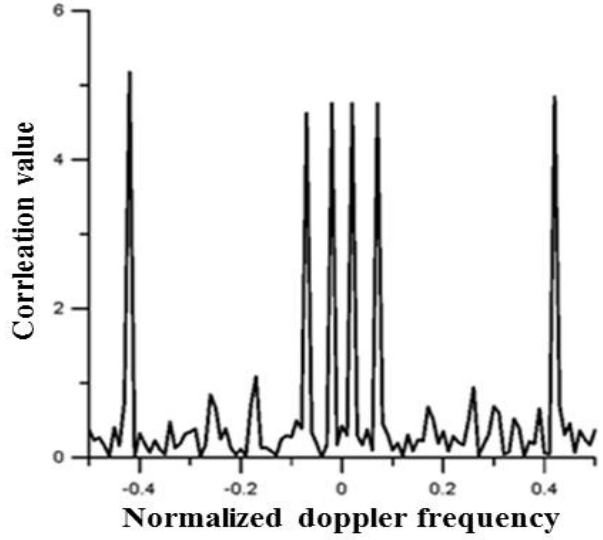

$\mathbf{N}=120$

Fig. 5. The estimation of normalized Doppler frequency in according to the number of antenna at the BS 
If pedestrian with mobile device is moving at $\pm 3 \mathrm{~km} / \mathrm{h}$, the Doppler freuqnecy is $\pm 277 \mathrm{~Hz}$ and the normalized Doppler frequency by the subcarrier spacing is $\pm 0.02 \mathrm{~Hz}$. Also, if bicycle with mobile device is moving at the $\pm 10 \mathrm{~km} / \mathrm{h}$, the Doppler freuqnecy is $\pm 926 \mathrm{~Hz}$ and the normalized Doppler frequency by the subcarrier spacing is $\pm 0.07 \mathrm{~Hz}$. Finally, if automobile with mobile device is moving at the $\pm 60 \mathrm{~km} / \mathrm{h}$, the Doppler freuqnecy is $\pm 5556 \mathrm{~Hz}$ and the normalized Doppler frequency by the subcarrier spacing is $\pm 0.43 \mathrm{~Hz}$. Fig. 5 shows the results of the estimation for the normalized Doppler frequency. At Fig. 5, $N$ means the number of antenna at the BS, the $\mathrm{x}$-axis means normalized Doppler and y-axis means correlation value at the normalized Doppler freuqnecy. The ML scheme estimates the normalized Doppler frequency with high correlation value at the x-axis. At Fig. 5, if the number of antenna at the $\mathrm{BS}$ is 30 , there are $\mathrm{x}$-axis values with high correlation value compared to the $\mathrm{x}$-axis values such as $\pm 0.02, \pm 0.07, \pm 0.43$ corresponding to the assumed velocity. Contrastively, if the number of antenna at the BS is 120 , the $x$-axis values such as $\pm 0.02, \pm 0.07, \pm 0.43$ have remarkably high correlation value than other $\mathrm{x}$-axis values. The reliability for estimation of the Doppler frequency is increased in according to the number of antenna at the BS. The reason is that the required information for estimation of the Doppler frequency is increased in according to the number of antenna in the massive MIMO system. Therefore, it is possible to reliably estimate the Doppler frequency at the massive MIMO system.

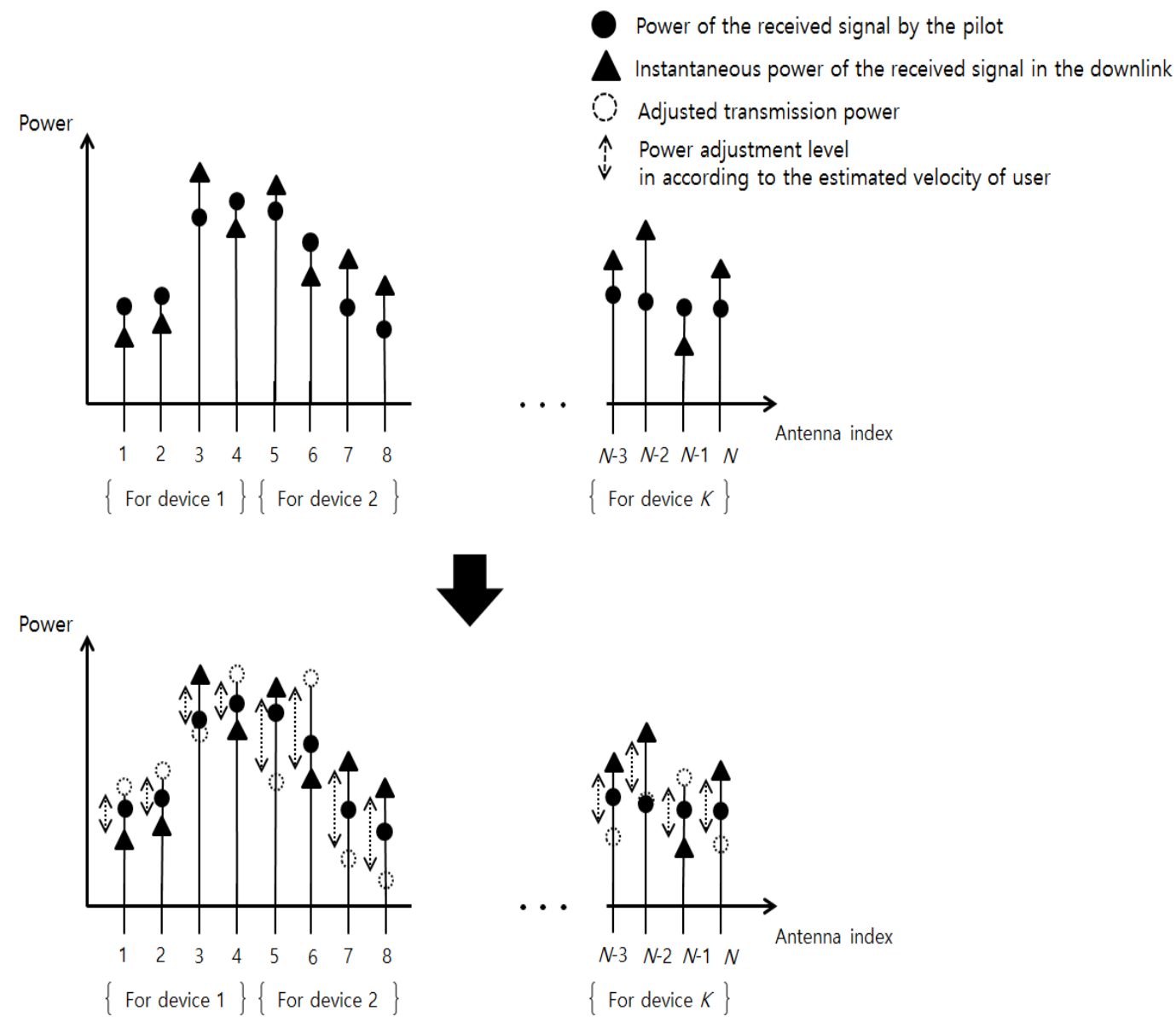

Fig. 6. The example for operation of the proposed adaptive power control 
The proposed adaptive power control estimates characteristics of the fast fading in according to the relative velocity using the estimated Doppler frequency. It is assumed that the system knows the fast fading models in according to the relative velocity such as Fig. $\mathbf{4}$ in advance. The system can estimate the velocity of mobile device using the Doppler frequency. Also, the system can estimate the characteristics of the fast fading for mobile device with the certain velocity using the estimated velcoity of mobile device. The proposed adaptive power control adjusts transmission power comparing the power of the received signal in the downlink and the power of the received signal by the pilot. If the power of the received signal in the downlink is larger than the power of the received signal by the pilot, transmission power is decreased. If the power of the received signal in the downlink is smaller than the power of the received signal by the pilot, transmission power is increased. When transmission power is adjusted, the adjustment level of power is determinded as the statistical average deviation of fast fading in according to the velocity. The adjustement level of power is calculated as follows,

$$
P_{A}(d B)=\frac{1}{N_{S}} \sum_{i=2}^{N_{S}}\left|F_{m}(i)-F_{m}(i-1)\right|
$$

where $N_{S}$ is the number of sample for fast fading model such as Fig. 4, $F_{m}$ is the loss value of each sample for fast pading model.

Fig. 6 shows the example of adjusted transmission power in according to the proposed adaptive power control. $N$ antennas of the BS is distributed to $K$ devices. For simple explanation, the device 1 , the device 2 , and the device $K$ are assumed to be moving at $3 \mathrm{~km} / \mathrm{h}$, $10 \mathrm{~km} / \mathrm{h}$, and $60 \mathrm{~km} / \mathrm{h}$ at Fig. 6. As shown at Fig. 6, as the comparison of the instantaneous power of the received signal and the power of the received signal by the pilot, transmission power is adjusted. Also, the adjustement level of power is different in according to the estimated relative velocity of the each device.

\section{Experimental Result and Evaluation of the Proposed Scheme}

For the performance evaluation of the proposed scheme, SINR is considered as evaluation criterion. Fig. 7 shows SINR of the proposed scheme and the conventional schemes. For the simulation, the three communication environments are considered. First, the environment is assumed that there are pedestrian $(3 \mathrm{~km} / \mathrm{h})$, bicycle $(10 \mathrm{~km} / \mathrm{h})$, and automobile $(10 \mathrm{~km} / \mathrm{h})$ as the ratio of $8: 1: 1$. Also, the suburb environment is assumed that there are pedestrian, bicycle, and automobile as the ratio of 2:2:6. Finally, the city environment is assumed that there are pedestrian, bicycle, and automobile as the ratio of 1:1:8. The conventional power control doesn't consider mobility of device and uses the constant adjustment level of power.

The experimental results show that the proposed power control scheme has about from $2 \mathrm{~dB}$ to $5 \mathrm{~dB}$ higher SINR than the conventional power control scheme in according to the number of mobile device and the communication environments. Especially, as the increase of rate for mobile device with fast velocity in the communication environment, the improvement of performance by the proposed power control scheme is remarkable.

As adavantage for increased SINR in the system, high-order modulation scheme can be used for throughput. Generally, modulation schemes with high throughput such as high-order 
QAM have adjacent constellation point. Therefore, if modulation scheme with high throughput is used for communication system with low SINR, the reliability is drmatically lowered. As a result of simulation, the proposed adaptive power control achieves high throughput of system in a mobile communication. Also, because the system using the modulation scheme with high throughput has high reliability at high SINR, the massive MIMO system can be effectively performed by the proposed adaptive power control in the mobile communication.

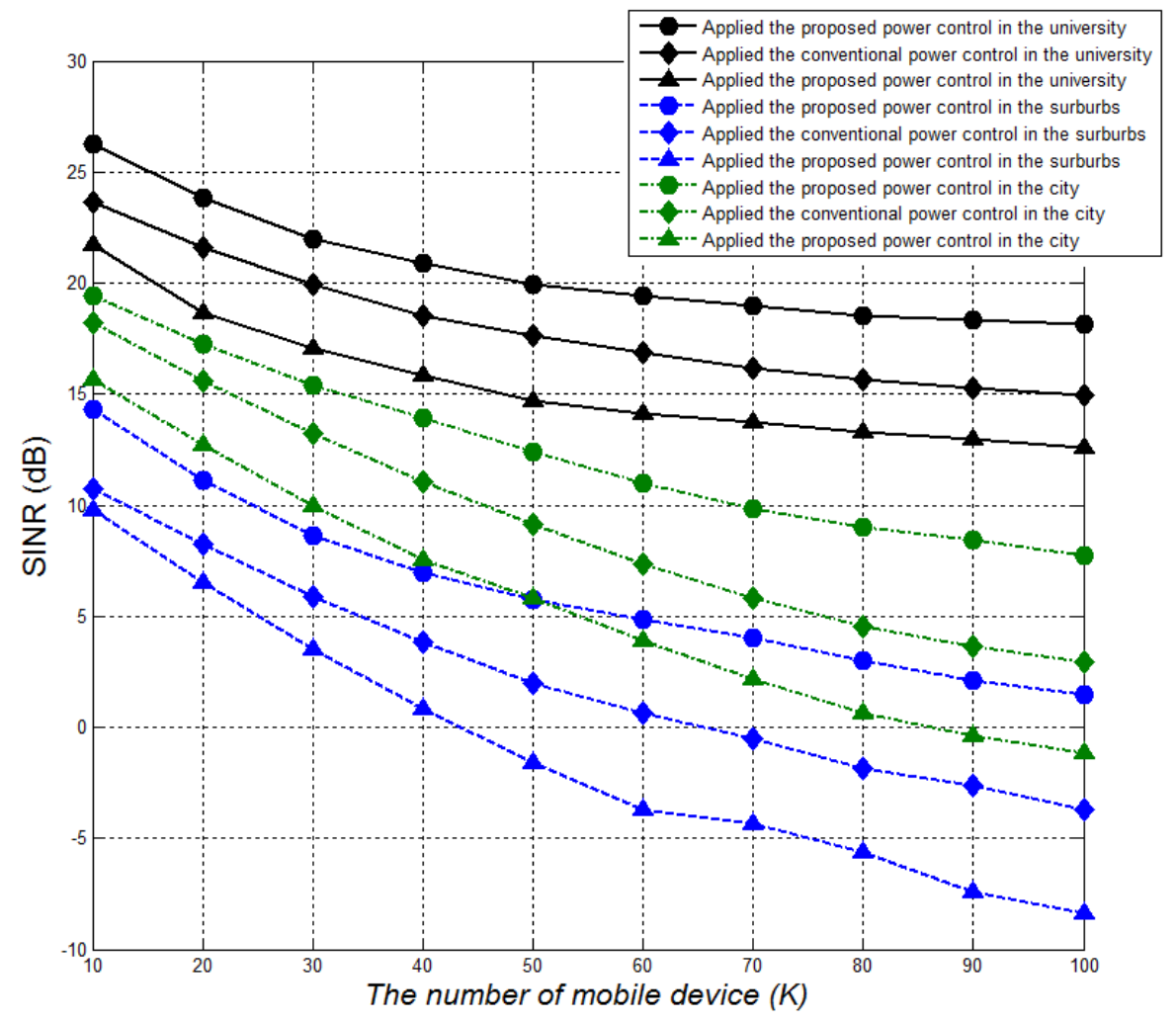

Fig. 7. The experimental results of the proposed scheme and conventional scheme

\section{Conclusion}

For the proportionally increased throughput in according to the number of antenna than the convnentional MIMO systems, the massive MIMO system using large scale antenna is emerging. The massive MIMO system effectively compensates channel between the BS and device and cancels interference signals from other antenna. The massive MIMO system uses TDD as duplexing scheme for reduction of the increased overhead by large scale antenna. Therefore, the massive MIMO system compensates the channel using the estimated channel information by pilots during the coherence time. However, the power of the received signal is continually flcutated by the mobility of device. As a result, the channel compensation of the massive MIMO system is non-perfect.

For enhancement of channel compensation of the massive MIMO system, the adaptive power control using the mobility of device is proposed. The proposed power control uses the relationship between the Doppler frequency and fast fading by the mobility of device. 
Therefore, the fast fading model in according to the mobility can be estimated using the relationship. The adjustment level of power is determined as the statistical average deviation of the estimated fast fading model in according to the mobility.

From the experimental result of the proposed power control, it is confirmed that the system has adaptively high SINR in according to the communication environment. Because SINR is decreased in according to the number of device, each base station can accommodate more devices by the proposed scheme in the network by unit of cell. Also, because the system using the modulation scheme with high throughput has high reliability at high SINR, the massive MIMO system can be effectively performed by the proposed adaptive power control in the mobile communication.

\section{References}

[1] S. Talwar, D. Choudhury, K. Dimou, E. Aryafar, B. Bangerter and K. Stewart, "Enabling technologies and architectures for 5G wireless," in IEEE Microwave Symposium (IMS), pp. 1-4, 2014. Article (CrossRef Link).

[2] H. Congzheng, T. Harrold, S. Armour and more authors, "Green radio: radio techniques to enable energy-efficient wireless networks," IEEE Communication Magazine, vol. 49, no. 6, pp. 46-54, 2011. Article (CrossRef Link).

[3] G. J. Foschini and M. J. Gans, "On limits of wireless communications in a fading environment when using multiple antennas," Wireless Personal Communications, vol. 6, no. 3, pp. 311-335, 1998. Article (CrossRef Link).

[4] G. J. Foschini, "Layered space-time architecture for wireless communication in a fading environment when using multi-element antennas," Bell Labs Technical Journal, vol. 1, no. 2, pp. 41-59, 1996. Article (CrossRef Link).

[5] P. W. Wolniansky, G. J. Foschini, G. D. Golden and R. A. Valenzuela, "V-BLAST: an architecture for realizing very high data rates over the rich-scattering wireless channel," in ISSSE98, pp. 295-300, 1998. Article (CrossRef Link).

[6] G. Redieteab, L. Cariou, P. Christin and J.-F. Helard, "SU/MU-MIMO in IEEE 802.11ac: PHY+MAC performance comparison for single antenna stations," in Wireless Telecommunications Symposium (WTS), pp. 1-5, April 18-20, 2012. Article (CrossRef Link).

[7] Y. Mehmood, W. Afzal, F. Ahmad, U. Younas, I. Rashid and I. Mehmood, "Large scaled multi-user MIMO system so called massive MIMO systems for future wireless communication networks," in Automation and Computing (ICAC), pp. 1-4, 2013. Article (CrossRef Link).

[8] E. Larsson, O. Edfors, F. Tufvesson and T. Marzetta, "Massive MIMO for next generation wireless systems," IEEE Communications Magazine, vol. 52, no. 2, pp. 186-195, 2014. Article (CrossRef Link).

[9] C.-W. Lee, C.-B. Chae, T.-H. Kim, S.-Y. Choi and J.-H. Lee, "Network massive MIMO for cell-boundary users: from a precoding normalization perspective," in IEEE Globecom Workshops, pp. 233-237, 2012. Article (CrossRef Link).

[10] H. Q. Ngo, E. G. Larsson and T. L. Marzetta, "Energy and spectral efficiency of very large multiuser MIMO systems," IEEE Transactions on Communications, vol. 61, no. 4, pp. 1436-1449, 2013. Article (CrossRef Link).

[11] J.-K. Ahn, H.-W. Jang and H.-K. Song, "An improved low complexity detection scheme in MIMO-OFDM systems," IEICE Transactions on Information and Systems, vol. E97.D, pp. 1336-1339, 2014. Article (CrossRef Link).

[12] H.-J. Choi, H.-K. Song, "Advanced QRD-M detection with iterative scheme in the MIMO system," IEICE Transactions on Information and Systems, vol. E97.D, pp. 340-343, 2014. Article (CrossRef Link). 
[13] H.-Y. Jeong and H.-K. Song, "Adaptive combining detection scheme using channel state in MIMO-OFDM system," IEICE Transactions on Fundamentals of Electronics and Computer Sciences, vol. E96.A, pp. 1905-1908, 2013. Article (CrossRef Link).

[14] H.-J. Park, M.-S. Baek and H.-K. Song, "Efficient signal detection technique for interactive digital broadcasting system with multiple antennas," IEEE Transactions on Consumer Electronics, vol. 58, no.2, pp. 293-301, 2012. Article (CrossRef Link).

[15] R. V. Nee and R. Prasad, "OFDM for wireless multimedia communications," Artech House, 2000. Article (CrossRef Link).

[16] S. Rajiv and J. Hem, "OFDM and its major concerns: a study with way out," IETE Journal of Education, vol. 54, no.1, pp. 26-49, 2013. Article (CrossRef Link).

[17] LTE; evolved universal terrestrial radio access (E-UTRA); radio frequency (RF) requirements for LTE pico node B (3GPP TR 36.931 version 9.0.0 Release 9), 2011. Article (CrossRef Link).

[18] J. Jose, A. Ashikhmin, T. L. Marzetta and S. Vishwanath, "Pilot contamination problem in multi-cell TDD systems," in IEEE ISIT, pp. 2184-2188, 2009. Article (CrossRef Link).

[19] T. E. Bogale and B. L. Long, "Pilot optimization and channel estimation for multiuser massive MIMO systems,” in IEEE CISS, pp. 1-6, 2014. Article (CrossRef Link).

[20] A. Ashikhmin and T. L. Marzetta, "Pilot contamination precoding in multi-cell large scale antenna systems," in IEEE ISIT, pp. 1137-1141, 2012. Article (CrossRef Link).

[21] F. Gini, M. Greco and A. Farina, "Multiple radar targets estimation by exploiting induced amplitude modulation," IEEE Transactions on Aerospace and Electronic Systems, vol. 39, no. 4, pp. 1316-1332, 2003. Article (CrossRef Link).

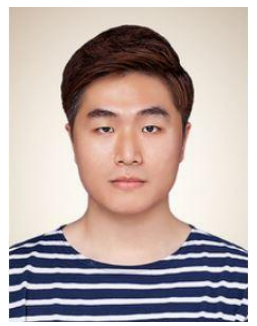

Chang-Bin Han received B.S. degree in Information \& Communication Engineering, Sejong University, Seoul, Korea, in 2014. He is working toward to M.S. degree in the Department of information and communications engineering, Sejong University, Seoul, Korea. His research interests are in the areas of wireless communication system design and MIMO detection.

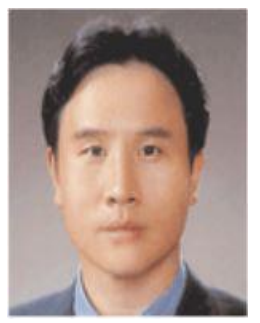

Byung-Jun Jang received B.S., M.S., and Ph.D. degrees in electronic engineering from Yonsei University, Seoul, Korea, in 1990, 1992, and 1997, respectively. From 1995 to 1999, he was with LG Electronics, Seoul, where he developed code-division multiple access and digital enhanced cordless telecommunication RF modules. From 1999 to 2005, he was with the Electronics and Telecommunications Research Institute, Daejeon, Korea, where he performed research in the fields of satellite RF components and monolithic microwave integrated circuits. In 2005, he joined Kookmin University, Seoul, where he is currently with the Department of Electrical Engineering. He is currently interested in the areas of RF circuit design and system analysis, radio-frequency-identification interference modeling, and biosensor design.

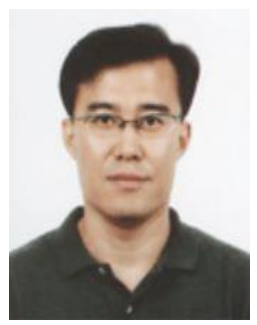

Hyoung-Kyu Song received B.S., M.S., and Ph.D. degrees in electronic engineering from Yonsei University, Seoul, Korea, in 1990, 1992, and 1996, respectively. From 1996 to 2000 he had been managerial engineer in Korea Electronics Technology Institute (KETI), Korea. Since 2000, he has been a professor of the Department of information and communications engineering, Sejong University, Seoul, Korea. His research interests include digital and data communications, information theory and their applications with an emphasis on mobile communications. 